\title{
Compressed Measurements Based Spectrum Sensing for Wideband Cognitive Radio Systems
}

\author{
Taha A. Khalaf, ${ }^{1,2}$ Mohammed Y. Abdelsadek, ${ }^{2}$ and Mohammed Farrag ${ }^{2,3}$ \\ ${ }^{1}$ Department of Electrical Engineering, University of Tabuk, Tabuk 71491, Saudi Arabia \\ ${ }^{2}$ Department of Electrical Engineering, Assiut University, Assiut 71516, Egypt \\ ${ }^{3}$ Electrical Engineering Department, King Khalid University, Abha 62529, Saudi Arabia \\ Correspondence should be addressed to Taha A. Khalaf; takhalaf@ut.edu.sa
}

Received 4 August 2015; Revised 8 December 2015; Accepted 10 December 2015

Academic Editor: Stefano Selleri

Copyright (C) 2015 Taha A. Khalaf et al. This is an open access article distributed under the Creative Commons Attribution License, which permits unrestricted use, distribution, and reproduction in any medium, provided the original work is properly cited.

\begin{abstract}
Spectrum sensing is the most important component in the cognitive radio (CR) technology. Spectrum sensing has considerable technical challenges, especially in wideband systems where higher sampling rates are required which increases the complexity and the power consumption of the hardware circuits. Compressive sensing (CS) is successfully deployed to solve this problem. Although CS solves the higher sampling rate problem, it does not reduce complexity to a large extent. Spectrum sensing via CS technique is performed in three steps: sensing compressed measurements, reconstructing the Nyquist rate signal, and performing spectrum sensing on the reconstructed signal. Compressed detectors perform spectrum sensing from the compressed measurements skipping the reconstruction step which is the most complex step in CS. In this paper, we propose a novel compressed detector using energy detection technique on compressed measurements sensed by the discrete cosine transform (DCT) matrix. The proposed algorithm not only reduces the computational complexity but also provides a better performance than the traditional energy detector and the traditional compressed detector in terms of the receiver operating characteristics. We also derive closed form expressions for the false alarm and detection probabilities. Numerical results show that the analytical expressions coincide with the exact probabilities obtained from simulations.
\end{abstract}

\section{Introduction}

There is a remarkable growing demand on wireless devices and services that use the electromagnetic spectrum for communication. In static license regime, the spectrum bands are assigned to licensed holders on a long term basis for large geographical regions. However, a large portion of the assigned spectrum remains underutilized [1]. Cognitive radio (CR) can utilize the spectrum more efficiently in an opportunistic fashion. CR allows a secondary user (SU) to use a specific spectrum band as long as its licensed primary user (PU) is protected against harmful interference. The Federal Communications Commission (FCC) defined the CR as follows: a radio or system that senses its operational electromagnetic environment and can dynamically and autonomously adjust its radio operating parameters to modify system operation [2]. The main functions for CRs are spectrum sensing, spectrum management, spectrum mobility, and spectrum sharing [3].
One key component of the CR system is spectrum sensing (SS), by which a SU radio can detect the presence or absence of the PUs and identify the available white spaces in the spectrum [4]. In order not to affect the performance of the PU, the SS process has to meet the sensing speed and accuracy requirements set by the FCC [2]. The problem becomes more challenging, particularly in wideband systems where the sampling rate has to be higher than or equal to the Nyquist rate. Consequently, the complexity and the cost of the hardware circuits as well as the power consumption would be high [5-7]. Moreover, the timing requirements for rapid sensing may only allow the acquisition of a small number of samples which may not provide accurate information about the existence of the PU.

Recently, compressive sensing (CS) is used to solve the high sampling rates problem in the CR systems. CS allows the sensing of sparse signals at sub-Nyquist sampling rates and a reliable recovery of the signal via computationally efficient 
algorithms $[8,9]$. In CS, the signal can be recovered from a small number of projections over a sensing basis (measurements) as long as it is sparse over the representation basis that is incoherent with the sensing basis. Examples of the sensing basis matrices are Gaussian, Bernoulli, partial Hadamard, and partial Fourier matrices. In order to reconstruct the signal, the sensing basis should satisfy the restricted isometry property (RIP) [10]. Signal reconstruction is the solution of an $l_{1}$-norm optimization problem to recover the high dimensional data from the low dimensional measurements.

In previous work of using CS in CR applications, SS is completed in three steps: sampling using an analog to information converter (AIC) at a sub-Nyquist rate, reconstructing the Nyquist rate signal or reconstructing the PU frequency response, and then applying one of the spectrum detection techniques on the reconstructed signal $[11,12,16]$. The SS process can be considered as a binary hypothesis test, where the detection algorithm has to decide which one of the two hypotheses is probably true. The mostly used detection schemes are matched filter detection [13], energy detection [14], and feature detection [15]. Although the algorithms proposed in $[11,12,16]$ reduce the hardware complexity of acquiring the signal, they increase the computational complexity through the reconstruction process. The fundamental task of SS is to detect the presence of the PUs. Therefore, the full reconstruction of the wideband signal is not necessary as long as the PU existence can be accurately detected from the compressed measurements. In [17], it was shown that the detection and estimation problem can be solved using the compressed measurements without reconstructing the original signal. However, the observation matrix chosen is the random observation matrix which serves the purpose of the reconstruction process not the detection process. The authors showed that the detection probability increases as the number of the compressed measurements increases. The analytical results of [17] show that the traditional detector outperforms the compressed measurements based detection algorithm in terms of the detection probability and the proposed detection algorithm will provide the same performance as the traditional detector only when the number of the compressed measurements equals the number of Nyquist rate samples.

In this paper, we propose an algorithm that enables the detection of the PU existence from the compressed measurements directly without going into the intermediate process of reconstructing the signal. This can be realized by designing a compressive sensing process that guarantees that the information used in the detection process is preserved in the compressed measurements. To achieve this, the sensing matrix has to be designed with constraints different from those used in the conventional CS [10] and the detector proposed in [17]. The algorithm we propose in this paper is based on the well-known energy detection techniques. Therefore, the sensing matrix should preserve the energy of PU signal. The discrete cosine transform (DCT) [18] has the property of energy compaction where most of the energy of the time domain signal will be concentrated in few samples of the DCT domain signal. Therefore, we adopt the DCT matrix as the sensing matrix in our algorithm. In addition to the reduction in the computational complexity due to the elimination of the reconstruction process, the results show that the proposed algorithm provides a better performance than the traditional energy detector that uses the Nyquist rate signal and accordingly the compressed detector proposed in [17]. Moreover, the detection algorithm we propose does not require a priori knowledge or estimation of the $\mathrm{PU}$ signal sparsity. We also derived closed form expressions for the detection and false alarm probabilities. Simulation results are provided to validate the derived expressions.

The remainder of this paper is organized as follows. The system model and the detection algorithms are described in Section 2. The derivation of the false alarm and detection probabilities is provided in Section 3. Section 4 presents the numerical results and discussions. Finally, the conclusions are drawn in Section 5.

\section{System Model and Detection Algorithms}

In this section, we present the system model, compressive sensing based energy detection (CSBED) algorithm, and the compressed measurements based energy detection (CMBED) algorithm. Let us first summarize some notations and definitions that will be used through the paper:

(i) $\mathscr{H}_{0}$ : it is the null hypothesis which states that there is no transmission from the PU in the band under consideration,

(ii) $\mathscr{H}_{1}$ : it is the alternative hypothesis which states that there is a transmission from the PU in the band under consideration,

(iii) $\mathscr{D}_{0}$ : the SU accepts $\mathscr{H}_{0}$ after applying the detection algorithm,

(iv) $\mathscr{D}_{1}$ : the SU rejects $\mathscr{H}_{0}$ after applying the detection algorithm,

(v) $P\left(\mathscr{D}_{1} \mid \mathscr{H}_{0}\right)$ : it is the probability that the SU decides that the PU exists while it does not (probability of false alarm).

2.1. System Model. In this paper, the primary network is assumed to be the television broadcasting system. According to the IEEE 802.22 WRAN standards [19], the PU signal is transmitted using orthogonal frequency division multiplexing (OFDM) with the following specifications: $54 \mathrm{MHz}$ : main carrier frequency, $4.45 \mathrm{Mbps}$ : data rate, 16-QAM: payload modulation, and 2048 FFT: size. The channel is modeled as an additive white Gaussian noise (AWGN) channel. The hypothesis model is defined as follows:

$$
\begin{aligned}
& \mathscr{H}_{0}: x(t)=n(t), \\
& \mathscr{H}_{1}: x(t)=s(t)+n(t),
\end{aligned}
$$

where $x(t)$ is the signal received by the SU, $s(t)$ is the PU signal with bandwidth $W$, and $n(t)$ is an (AWGN) with zero mean and one-sided power spectral density of $N_{01}$. The received signal is sensed using CS to get the low dimensional data vector $\mathbf{y}$ which has a sub-Nyquist sampling rate and is given by

$$
\mathbf{y}=\Phi \mathbf{x}
$$




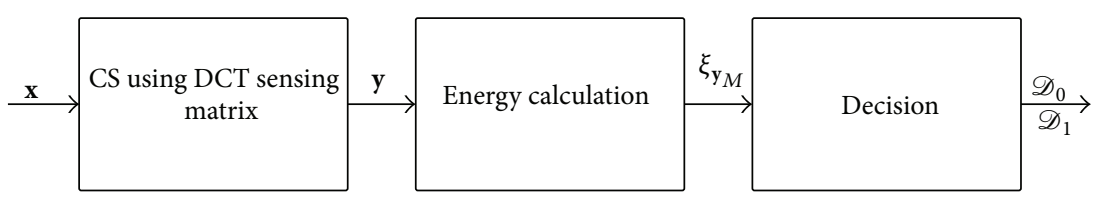

FIGURE 1: Block diagram of the CMBED algorithm.

TABLE 1: Complexities and memory requirements for some OMP computational approaches [24].

\begin{tabular}{lcc}
\hline Algorithm & Complexity & Memory \\
\hline Naive way & $N M+M k+M k^{2}+k^{3}$ & $M N$ \\
Cholesky & $N M+M k+k^{2}$ & $N^{2}+N M+k+k^{2}$ \\
$\begin{array}{l}\text { Decomposition-1 } \\
\text { Cholesky }\end{array}$ & $N k+k^{2}$ & $N^{2}+N M+k+k^{2}$ \\
$\begin{array}{l}\text { Decomposition-2 } \\
\text { QR }\end{array}$ & $N M+M k$; solve: $K^{2}$ & $N M+M k+k^{2}$ \\
$\begin{array}{l}\text { Decomposition-1 } \\
\text { QR }\end{array}$ & $N k+M k+k^{2}$ & $N^{2}+N M+M k+k^{2}$ \\
$\begin{array}{l}\text { Decomposition-2 } \\
\text { Matrix Inversion }\end{array}$ & $N k+M k$ & $N^{2}+N M+M k$ \\
Lemma & & \\
\hline
\end{tabular}

where $\mathbf{x}$ is an $N \times 1$ vector which represents the Nyquist rate samples of $x(t), \mathbf{y}$ is the $M \times 1$ compressed measurements vector, and $\Phi$ is an $M \times N$ sensing matrix $(M<N)$.

\subsection{Compressive Sensing Based Energy Detection Algorithm} (CSBED). In CSBED, the Nyquist rate signal is reconstructed from the compressed measurements vector $y$ of (3) and then the energy detector is applied to the reconstructed signal. The reconstruction process is a linear inverse problem with sparseness constraint. It was shown that this problem is an NP-hard [20]. Basis Pursuit (BP) [21] is a reconstruction technique that transforms the problem to a convex optimization problem that can be solved by linear programming as follows:

$$
\begin{aligned}
\widehat{\mathbf{x}}=\arg \min _{\mathbf{x}} & \|\mathbf{x}\|_{1} \\
\text { s.t. } & \mathbf{y}=\boldsymbol{\Phi} \mathbf{x} .
\end{aligned}
$$

Several reconstruction techniques have been proposed in the literature. Examples of these techniques are Matching Pursuit (MP) [22] and Orthogonal Matching Pursuit (OMP) [23]. These techniques differ in the computational complexity and memory requirements. For instance, the computational complexity and memory requirements of the OMP algorithms as a function of the iteration number $k$ are summarized in Table 1. In QR-1, only after the final iteration $K$ does OMP find the solution [24]. The accuracy of the reconstructed signal depends on the number of compressed measurements $M$ and the number of iterations. In order to detect the existence of the PU, the energy of the reconstructed signal $\xi_{\widehat{\mathrm{x}}}$ is calculated and compared with a predetermined threshold $\lambda$ as follows:

$$
\xi_{\widehat{\mathbf{x}}} \underset{\mathscr{D}_{1}}{\stackrel{\mathscr{D}_{0}}{\lessgtr}} \lambda .
$$

2.3. Compressed Measurements Based Energy Detection Algorithm (CMBED). In this paper, we propose to detect the existence of the PU directly from the compressed measurements without reconstructing the Nyquist rate signal. Hence, the computational complexity will be dramatically reduced. Figure 1 shows the block diagram of the proposed detection algorithm. In ordinary CS, the sensing matrix is chosen with specific constraints such that the Nyquist rate signal can be accurately reconstructed. Since the Nyquist rate signal will not be reconstructed in the proposed algorithm, the constraints of the sensing matrix are different. The sensing matrix has to preserve specific parameters of the signal such that the PU can be detected. In this paper, the energy detector will be applied to the compressed measurements. Therefore, the sensing matrix has to preserve the energy of PU signal. The discrete cosine transform (DCT) expresses a finite sequence of data points in terms of a sum of cosine functions oscillating at different frequencies. One of the properties of the DCT is the strong energy compaction where most of the signal information tends to be concentrated in the few lowfrequency coefficients [18]. In the proposed algorithm, the CS is performed using the DCT sensing matrix. The entry of the sensing matrix that is located at the $k$ th row and the $i$ th column is given by

$$
\phi(k, i)=c(k) \cos \left(\frac{\pi(2 i+1) k}{2 N}\right),
$$

where $k=0,1, \ldots, M-1, i=0,1, \ldots, N-1$, and

$$
c(k)= \begin{cases}\sqrt{\frac{1}{N}}, & k=0, \\ \sqrt{\frac{2}{N}}, & k \neq 0 .\end{cases}
$$

It is worth mentioning that the first $M$ rows of the DCT matrix are chosen as they correspond to the high-energy coefficients. After CS, according to Parseval's theorem [18], the energy of the signal can be calculated from the compressed measurements as follows [25]:

$$
\xi_{\mathbf{y}_{M}}=\sum_{k=0}^{M-1}\left(\frac{y_{k}}{\sqrt{N_{01} W}}\right)^{2} .
$$

Afterwards, the energy detector decision variable $\xi_{\mathbf{y}_{M}}$ is compared with a predetermined threshold $\lambda$ to decide $\mathscr{D}_{0}$ or $\mathscr{D}_{1}$ as follows:

$$
\xi_{\mathbf{y}_{M}} \stackrel{\mathscr{D}_{0}}{\lessgtr} \lambda
$$




\section{False Alarm and Detection Probabilities}

In this section, we derive closed form expressions for the false alarm and detection probabilities of the proposed CMBED algorithm. The probability of false alarm $P_{F}$ is defined as the probability that the SU declares that the PU exists while it does not and it is given by

$$
P_{F}=P\left(\mathscr{D}_{1} \mid \mathscr{H}_{0}\right)=P\left(\xi_{\mathbf{y}_{M}} \geq \lambda \mid \mathscr{H}_{0}\right) .
$$

When the PU does not exist, the signal received by the SU is an AWGN as given by (1). Therefore, the output of CS would be a vector of $M$ Gaussian random variables (linear combination of i.i.d Gaussian RV's is a Gaussian RV) which is given by

$$
\mathbf{y}=\left[\begin{array}{llll}
v_{1} & v_{2} & \cdots & v_{M}
\end{array}\right]^{T},
$$

where $v_{1}, v_{2}, \ldots, v_{M}$ are independent and identically distributed Gaussian RV's each has zero mean and variance $N_{01} W$. Substituting from (11) into (8) yields

$$
\xi_{\mathbf{y}_{M}}=\sum_{k=0}^{M-1}\left(\frac{v_{k}}{\sqrt{N_{01} W}}\right)^{2} .
$$

The decision variable of (12) follows the central chi-square distribution with $M$ degrees of freedom and unity variance with the following probability density function [13, eq. (2.321)]:

$$
f_{H 0}\left(\xi_{\mathrm{y}_{M}}\right)=\frac{1}{2^{M / 2} \Gamma(M / 2)} \xi^{M / 2-1} e^{-\xi / 2}
$$

where $\Gamma(\cdot)$ is the gamma function. From (10) and (13), the probability of false alarm would be given by

$$
\begin{aligned}
P_{F} & =\frac{1}{2^{M / 2} \Gamma(M / 2)} \int_{\lambda}^{\infty} \xi^{M / 2-1} e^{-\xi / 2} \mathrm{~d} \xi \\
& =\frac{\Gamma(M / 2, \lambda / 2)}{\Gamma(M / 2)},
\end{aligned}
$$

where $\Gamma(\cdot, \cdot)$ is the upper incomplete gamma function.

The probability of detection is given by

$$
P_{D}=P\left(\mathscr{D}_{1} \mid \mathscr{H}_{1}\right)=P\left(\xi_{\mathrm{y}_{M}} \geq \lambda \mid \mathscr{H}_{1}\right) .
$$

When the PU exists, the signal received by the SU is the summation of the PU signal and a white Gaussian noise as given by (2). The output of CS would be

$$
\mathbf{y}=\left[\begin{array}{llll}
s_{1}+v_{1} & s_{2}+v_{2} & \cdots & s_{M}+v_{M}
\end{array}\right]^{T},
$$

where $s_{1}, s_{2}, \ldots, s_{M}$ are the $M$ DCT coefficients of the PU signal. Substituting from (16) into (8) yields

$$
\xi_{\mathrm{y}_{M}}=\sum_{k=0}^{M-1}\left(\frac{s_{k}+v_{k}}{\sqrt{N_{01} W}}\right)^{2} .
$$

The decision variable of (17) is a noncentral chi-square random variable with $M$ degrees of freedom and has the following probability density function [13, eq. (2.3-29)]

$$
f_{H 1}\left(\xi_{\mathrm{y}_{M}}\right)=\frac{1}{2}\left(\frac{\xi}{\mu}\right)^{M-2 / 4} e^{-(\xi+\mu) / 2} I_{M / 2-1}(\sqrt{\mu \xi}),
$$

where $I_{m}(\cdot)$ is the $m$ th order modified Bessel function of the first kind and $\mu$ is the noncentrality parameter which is given by [14]

$$
\mu=\sum_{k=0}^{M-1}\left(\frac{s_{k}}{\sqrt{N_{01} W}}\right)^{2} .
$$

Let $E_{s}=\sum_{k=0}^{N-1} s_{k}^{2}$ be the total energy of the PU signal and $\eta_{E}$ be the fraction of energy contained in the $M$ DCT coefficients relative to $E_{s}$ (i.e., $\eta_{E}=1$ when $M=N$ ). Then

$$
\eta_{E}=\frac{1}{E_{s}} \sum_{k=0}^{M-1} s_{k}^{2}
$$

Substituting from (20) into (19) yields

$$
\mu=\eta_{E} \frac{E_{s}}{N_{01} W}=\eta_{E} \gamma,
$$

where $\gamma$ is the signal to noise ratio. From (15), (18), and (21), the probability of detection can be calculated as follows:

$$
\begin{aligned}
P_{D} & =\frac{1}{2} \int_{\lambda}^{\infty}\left(\frac{\xi}{\mu}\right)^{(M-2) / 4} e^{-(\xi+\mu) / 2} I_{M / 2-1}(\sqrt{\mu \xi}) \mathrm{d} \xi \\
& =Q_{M / 2}\left(\sqrt{\eta_{E} \gamma}, \sqrt{\lambda}\right),
\end{aligned}
$$

where $Q_{M / 2}(\cdot, \cdot)$ is the generalized Marcum $Q$-function [13, eq. (2.3-36)]. The probability of misdetection is given by

$$
\begin{aligned}
P_{M} & =P\left(\mathscr{D}_{0} \mid \mathscr{H}_{1}\right)=1-P_{D} \\
& =1-Q_{M / 2}\left(\sqrt{\eta_{E} \gamma}, \sqrt{\lambda}\right) .
\end{aligned}
$$

The performance of the proposed algorithm is compared with that of the traditional energy detector (TED) and the compressed detector presented in [17] where the probability of detection is approximated by

$$
P_{D}=Q\left(Q^{-1}\left(P_{F}\right)-\sqrt{\frac{M}{N}} \sqrt{\gamma}\right) .
$$

In the compressed detector [17], decreasing the compression ratio (i.e., decreasing $M$ ) causes a degradation in performance but the algorithm we propose in this paper provides a better performance at low compression ratios as will be shown and intuitively interpreted in Section 4 .

\section{Numerical Results and Discussions}

In this section, we present numerical results to study the performance of the proposed sensing technique. Unless otherwise stated, the PU signal is assumed to follow the IEEE 


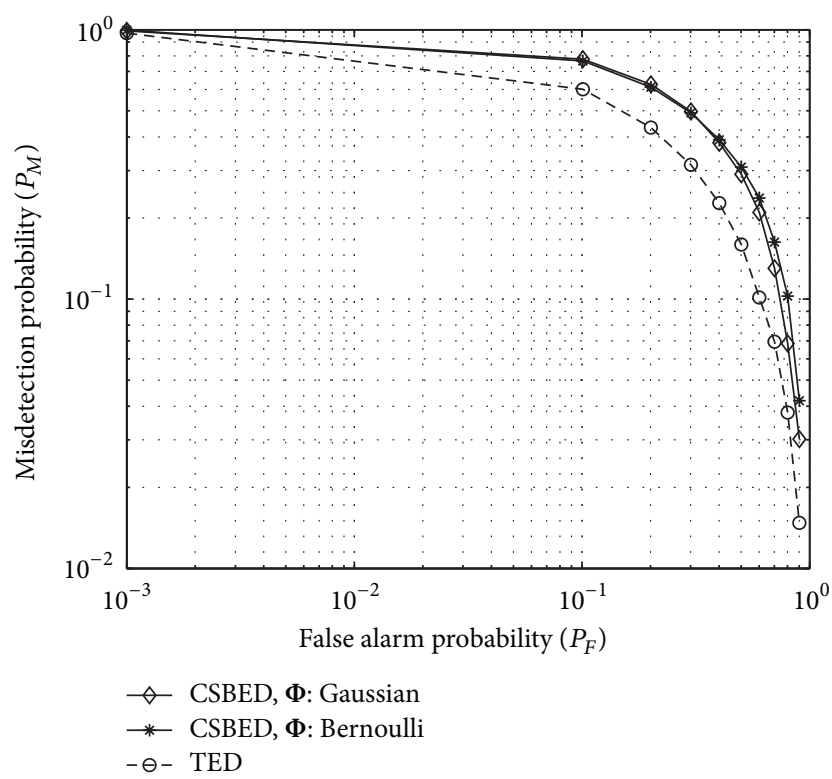

FIgURE 2: Complementary ROC when $N=512, M / N=70 \%$, and $\gamma=15 \mathrm{~dB}$.

802.22 WRAN standards described in Section 2.1. Figure 2 compares the complementary receiver operating characteristics (ROC) of the traditional energy detector (TED) (i.e., the energy detector is applied to signal that is sampled at a rate higher than the Nyquist rate) with that of the CSBED algorithm. Gaussian and Bernoulli matrices are used to perform the CS process. In this comparison, the compression ratio $(M / N)$ is $70 \%$ and the SNR equals $15 \mathrm{~dB}$. In CSBED, the OMP algorithm is used to reconstruct the signal. We find that the performance of the CSBED technique is worse than that of the TED. That is because the PU signal was not perfectly reconstructed. Therefore, the TED will be considered when evaluating the performance of the CMBED technique proposed in this paper.

Figure 3 compares the complementary ROC of the proposed CMBED algorithm with that of the TED algorithm, using compressed measurements sensed with Gaussian and partial Fourier ensemble sensing matrices, and the compressed detector in [17], when the compression ratio $(M / N)$ is $10 \%$ and the SNR equals $15 \mathrm{~dB}$. The false alarm and the misdetection probabilities are obtained from both simulations and the analytical results of Section 3. We find that the analytical results coincide with the simulation results which validates (14) and (22). We also find that the proposed CMBED algorithm provides a better performance than the TED algorithm and other algorithms. That is because the compressed measurements contain most of the PU signal energy and only a fraction of the noise energy. Figure 4 shows the percentage of the energy $\eta_{E}$ contained in $M$ compressed measurements of both $\mathrm{PU}$ and noise signals against the compression ratio $(M / N)$. We find that, at $M / N=10 \%$, the percentage of the energy contained in the compressed measurements of the PU signal equals $91.5 \%$ and that of the noise signal equals $10 \%$. That is because, on the one hand, the DCTbased CS preserves the energy of the PU signal, and, on

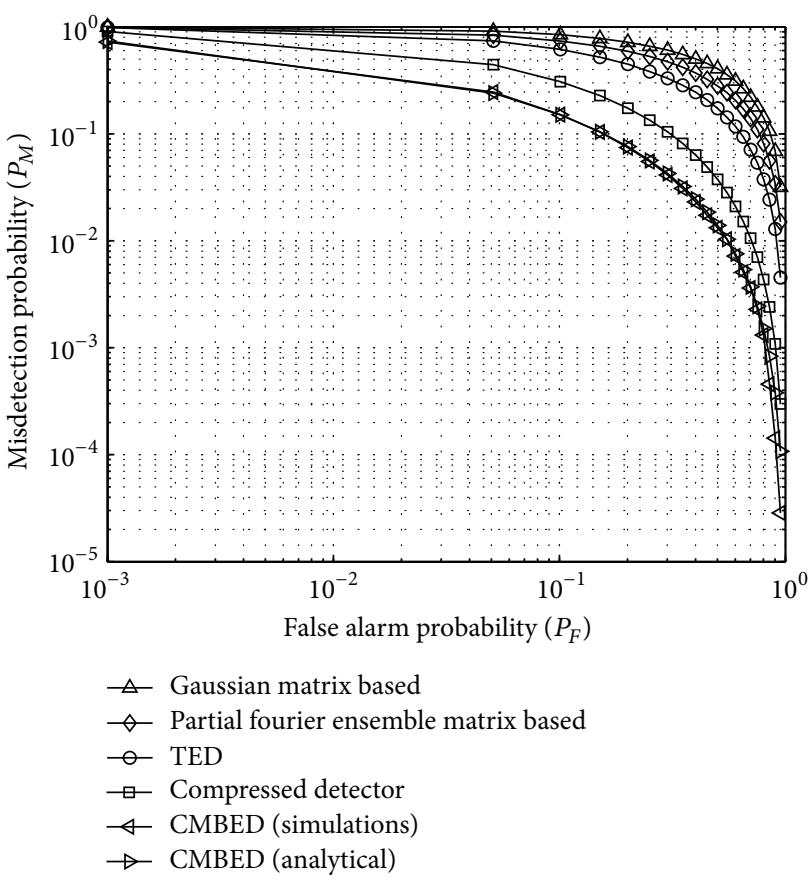

FIgURE 3: Complementary ROC when $N=512$ and $\gamma=15 \mathrm{~dB}$.

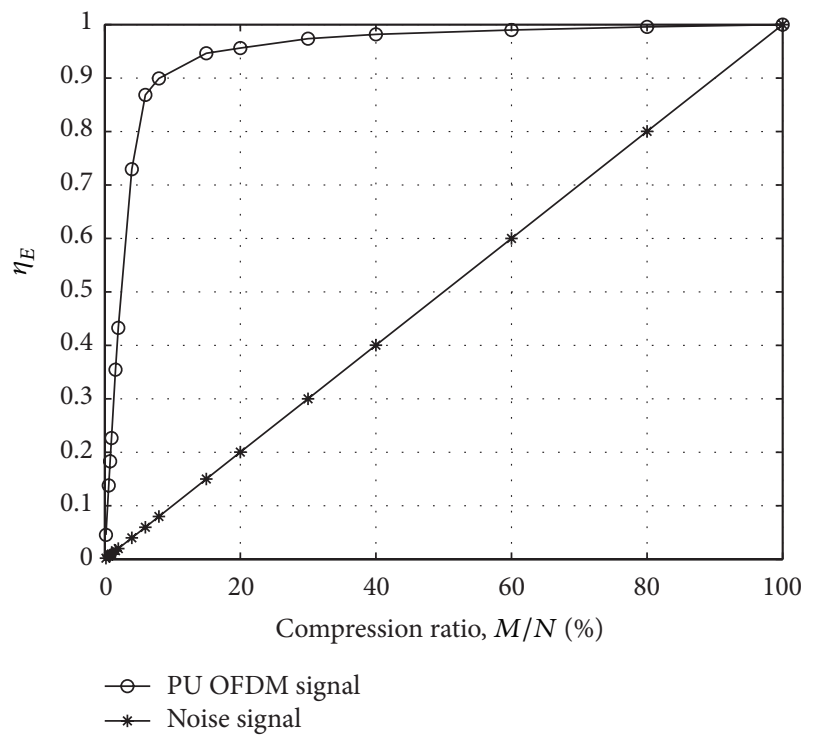

FIGURE 4: $\eta_{E}$ versus compression ratio for AWGN and OFDM signal.

the other hand, it would only preserve $M / N$ of the AWGN energy.

Figure 5 shows the detection probability $P_{D}$ against the compression ratio $M / N$ when $N=128, \gamma=15 \mathrm{~dB}$, and target $P_{F}=0.01$ for the proposed CMBED algorithm, TED, and compressed detector in [17]. We find that $P_{D}$ increases as $M / N$ increases until it reaches a maximum value of 0.903 at a compression ratio of $5.5 \%$ and then it decreases as $M / N$ increases. This convex behavior of the detection probability of the CMBED algorithm can be interpreted as follows. Let $E_{s_{M}}$ and $E_{n_{M}}$ denote the energies contained in 


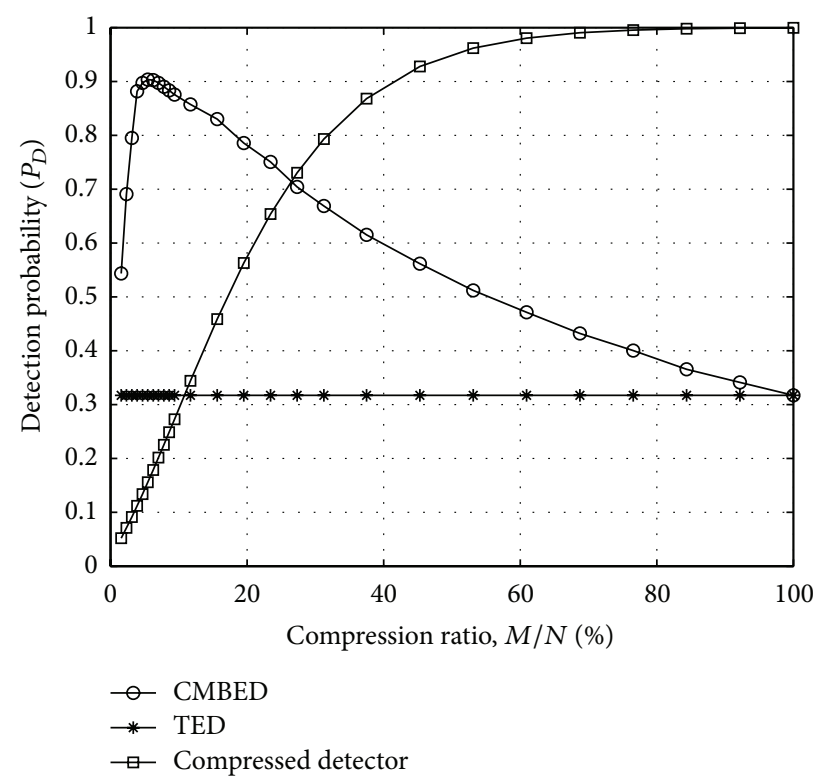

FIGURE 5: Probability of detection versus the compression ratio when $P_{F}=0.01, N=128$, and $\gamma=15 \mathrm{~dB}$.

the $M$ compressed measurements of the PU and the noise signals, respectively. It is clear that both $E_{s_{M}}$ and $E_{n_{M}}$ will increase as the compression ratio increases when $N$ is fixed. In order to keep the false alarm probability fixed (i.e., $P\left(E_{n_{M}}>\right.$ $\lambda)=0.01$ ), increasing the compression ratio (or equivalently increasing $M$ ) necessitates an increase in the threshold $\lambda$. The detection probability is defined as the probability that the energy contained in the compressed measurements is greater than the threshold when the PU exists (i.e., $P\left(E_{s_{M}}+E_{n_{M}}>\lambda\right)$ ). When $M$ increases, both sides of the inequality will increase but not with the same rate. At lower compression ratios (i.e., smaller values of $M$ ), the growth of $E_{s_{M}}$ with respect to $M$ is much higher than that of $E_{n_{M}}$ (as shown in Figure 4) and accordingly $\lambda$. Therefore, the detection probability increases as $M / N$ increases. At higher compression ratios, increasing $M$ results in a negligible increase in $E_{s_{M}}$; however, a considerable increase occurs in $\lambda$. Hence, the detection probability decreases as $M / N$ increases.

Figure 6 shows the detection probability against the signal-to-noise ratio for CMBED, TED, and the compressed detector in [17] when $P_{F}=0.01$ and compression ratio of $10 \%$ at $N=128$. We find that the detection probability of the proposed CMBED algorithm is higher than other algorithms.

\section{Conclusions}

In this paper, we proposed a spectrum sensing technique for wideband cognitive radio systems. In order to detect the existence of the PU, the SU firstly samples the received signal at a sub-Nyquist rate using compressive sensing and then applies the energy detector on the compressed measurements. Consequently, the complexity required in reconstructing the Nyquist rate signal is eliminated. The DCT sensing matrix was used for the compressive sensing step due to its energy compaction property. In addition to the considerable

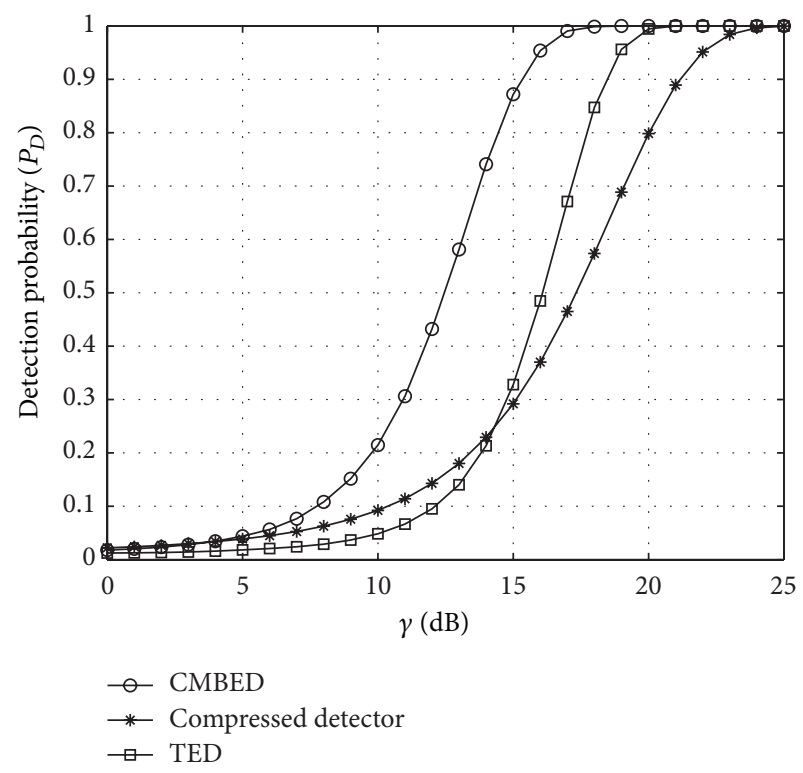

FIgURE 6: Probability of detection versus SNR when $P_{F}=0.01$ and a compression ratio of $10 \%$.

complexity reduction, the results showed that proposed algorithm provides a better detection probability compared to traditional schemes at the same false alarm probability. That is because although the compressed measurements contain most of the energy of the PU signal, they only contain a fraction of the energy of the noise signal. We also derived analytical expressions for the false alarm and misdetection probabilities. These results are validated using simulations.

\section{Conflict of Interests}

The authors declare that there is no conflict of interests regarding the publication of this paper.

\section{Acknowledgment}

The authors would like to acknowledge partial financial support for this work from the Deanship of Scientific Research (DSR), University of Tabuk, Tabuk, Saudi Arabia, under Grant no. S-1436-0216.

\section{References}

[1] B. Wang and K. J. Ray Liu, "Advances in cognitive radio networks: a survey," IEEE Journal on Selected Topics in Signal Processing, vol. 5, no. 1, pp. 5-23, 2011.

[2] Federal Communications Commission, "Notice of proposed rule making and order: facilitating opportunities for flexible, efficient, and reliable spectrum use employing cognitive radio technologies," ET Docket no. 03-108, Federal Communications Commission, 2005.

[3] I. F. Akyildiz, W.-Y. Lee, M. C. Vuran, and S. Mohanty, "Next generation/dynamic spectrum access/cognitive radio wireless networks: a survey," Computer Networks, vol. 50, no. 13, pp. 2127-2159, 2006. 
[4] N. Yadav and S. Rathi, "Spectrum sensing techniques: research, challenge and limitations," International Journal of Electronics \& Communication Technology, vol. 2, no. 4, pp. 240-245, 2011.

[5] F. Penna and R. Garello, "Detection of discontinuous signals for cognitive radio applications," IET Communications, vol. 5, no. 10, pp. 1453-1461, 2011.

[6] W. Li, C. Yang, Z. Wu, B. Tan, and Y. Fu, "Selective sensing and access strategy to maximize throughput in cognitive radio sensor network," International Journal of Distributed Sensor Networks, vol. 2014, Article ID 749739, 9 pages, 2014.

[7] L. Safatly, M. Bkassiny, M. Al-Husseini, and A. El-Hajj, "Cognitive radio transceivers: RF, spectrum sensing, and learning algorithms review," International Journal of Antennas and Propagation, vol. 2014, Article ID 548473, 21 pages, 2014.

[8] D. L. Donoho, "Compressed sensing," IEEE Transactions on Information Theory, vol. 52, no. 4, pp. 1289-1306, 2006.

[9] J. Haupt and R. Nowak, "Compressive sampling for signal detection," in Proceedings of the IEEE International Conference on Acoustics, Speech and Signal Processing (ICASSP '07), vol. 3, pp. 1509-1512, IEEE, Honolulu, Hawaii, USA, April 2007.

[10] E. J. Candes, J. Romberg, and T. Tao, "Robust uncertainty principles: exact signal reconstruction from highly incomplete frequency information," IEEE Transactions on Information Theory, vol. 52, no. 2, pp. 489-509, 2006.

[11] Z. Tian and G. B. Giannakis, "Compressed sensing for wideband cognitive radios," in Proceedings of the IEEE International Conference on Acoustics, Speech and Signal Processing (ICASSP '07), vol. 4, pp. IV1357-IV1360, Honolulu, Hawaii, USA, April 2007.

[12] Y. Wang, A. Pandharipande, Y. L. Poloy, and G. Leusy, "Distributed compressive wide-band spectrum sensing," in Proceedings of the Information Theory and Applications Workshop (ITA '09), pp. 178-183, IEEE, San Diego, Calif, USA, February 2009.

[13] J. G. Proakis and M. Salehi, Digital Communications, McGrawHill, Singapore, 5th edition, 2008.

[14] F. F. Digham, M.-S. Alouini, and M. K. Simon, "On the energy detection of unknown signals over fading channels," IEEE Transactions on Communications, vol. 55, no. 1, pp. 21-24, 2007.

[15] W.-L. Chin, H.-C. Kuo, and H.-H. Chen, "Features detection assisted spectrum sensing in wireless regional area network cognitive radio systems," IET Communications, vol. 6, no. 8, pp. 810-818, 2012.

[16] S. Hong, "Direct spectrum sensing from compressed measurements," in Proceedings of the IEEE Military Communications Conference (MILCOM '10), pp. 1187-1192, IEEE, San Jose, Calif, USA, November 2010.

[17] M. A. Davenport, M. B. Wakin, and R. G. Baraniuk, "Detection and estimation with compressive measurements," Tech. Rep., Department of ECE, Rice University, Houston, Tex, USA, 2006.

[18] A. V. Oppenheim, R. W. Schafer, and J. R. Buck, Discrete-Time Signal Processing, Prentice-Hall, Upper Saddle River, NJ, USA, 2nd edition, 1999.

[19] IEEE, "Part 22: cognitive wireless RAN Medium Access Control (MAC) and Physical Layer (PHY) specifications: polices and procedures for operation in the TV bands," IEEE Std. 802.22, 2011.

[20] E. Candes, M. Rudelson, T. Tao, and R. Vershynin, "Error correction via linear programming," in Proceedings of the 46th Annual IEEE Symposium on Foundations of Computer Science (FOCS '05), pp. 668-681, IEEE, Pittsburgh, Pa, USA, October 2005.
[21] S. S. Chen, D. L. Donoho, and M. A. Saunders, "Atomic decomposition by basis pursuit," SIAM Journal on Scientific Computing, vol. 20, no. 1, pp. 33-61, 1998.

[22] S. G. Mallat and Z. Zhang, "Matching pursuits with time-frequency dictionaries," IEEE Transactions on Signal Processing, vol. 41, no. 12, pp. 3397-3415, 1993.

[23] J. A. Tropp and A. C. Gilbert, "Signal recovery from random measurements via orthogonal matching pursuit," IEEE Transactions on Information Theory, vol. 53, no. 12, pp. 4655-4666, 2007.

[24] B. L. Sturm and M. G. Christensen, "Comparison of orthogonal matching pursuit implementations," in Proceedings of the 20th European Signal Processing Conference (EUSIPCO '12), pp. 220224, August 2012.

[25] H. Urkowitz, "Energy detection of unknown deterministic signals," Proceedings of the IEEE, vol. 55, no. 4, pp. 523-531, 1967. 

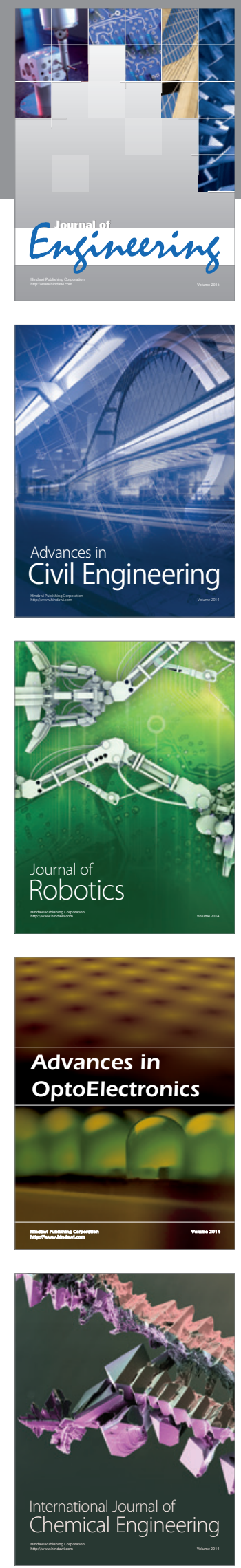

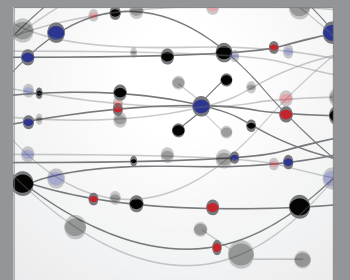

The Scientific World Journal
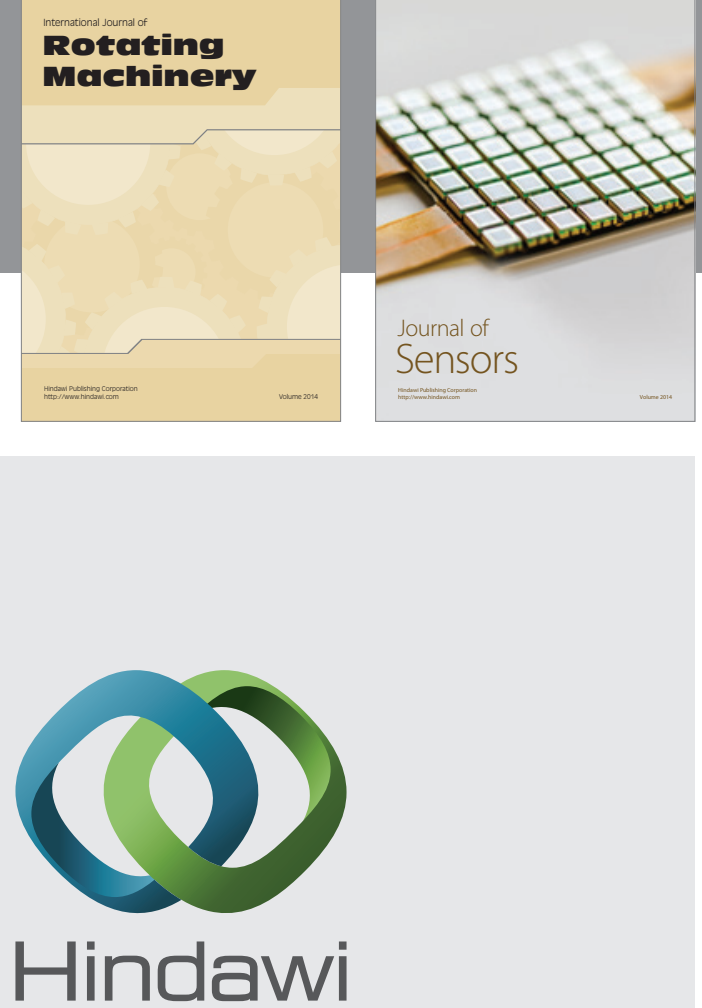

Submit your manuscripts at http://www.hindawi.com
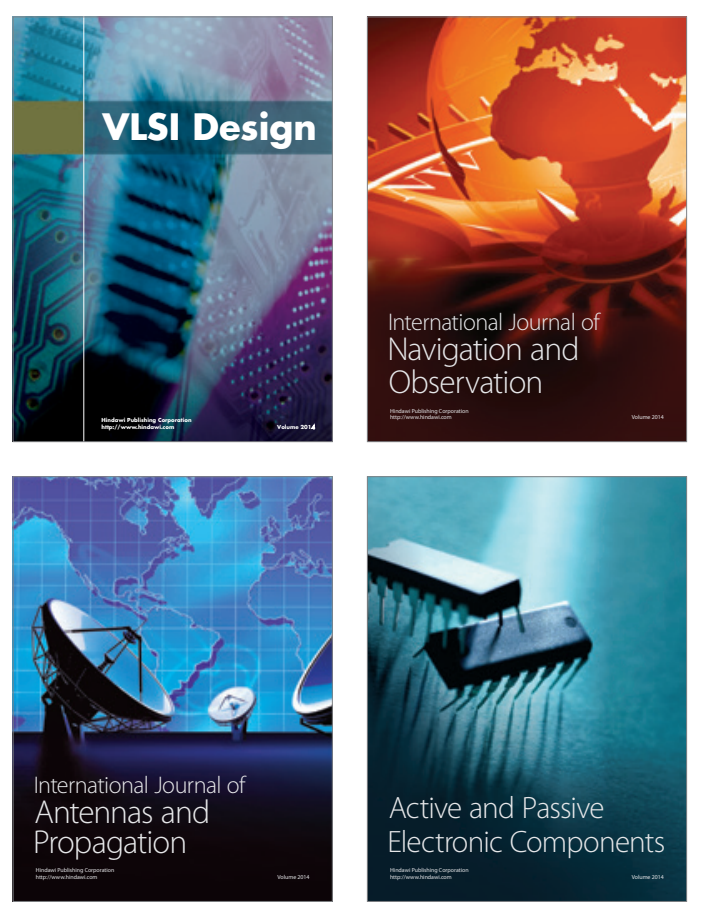
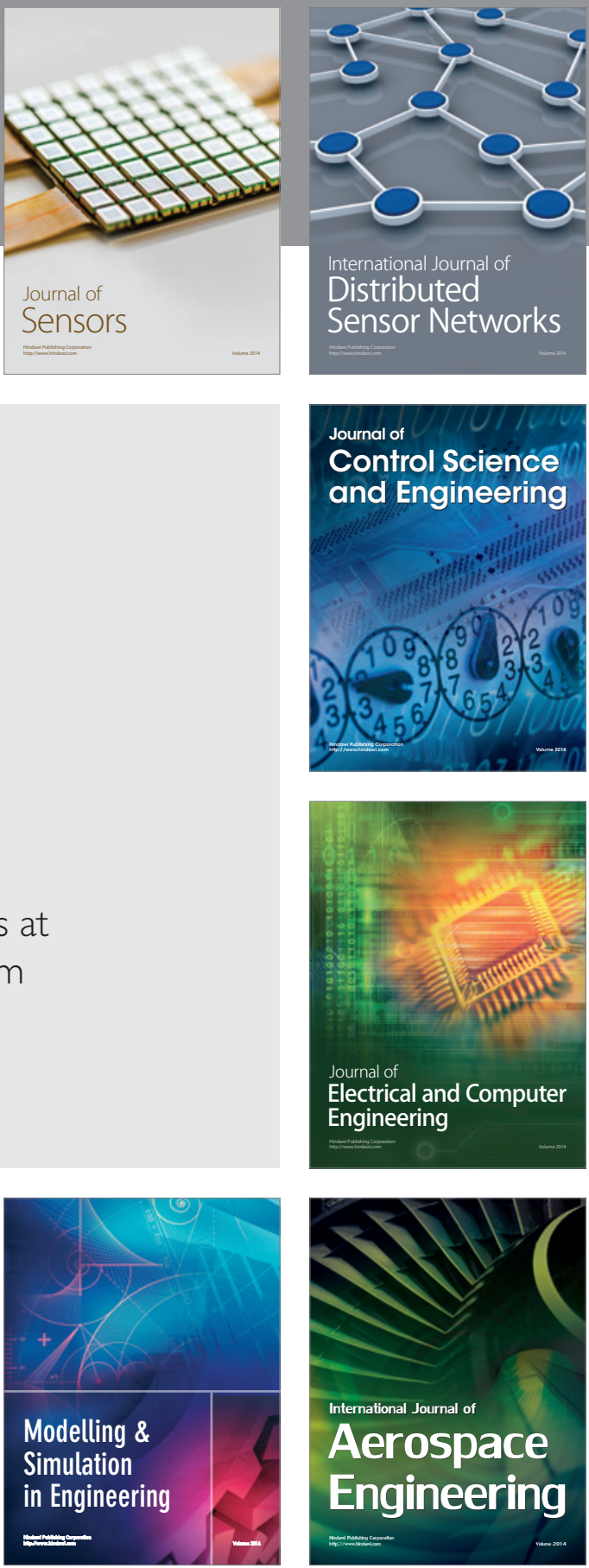

Journal of

Control Science

and Engineering
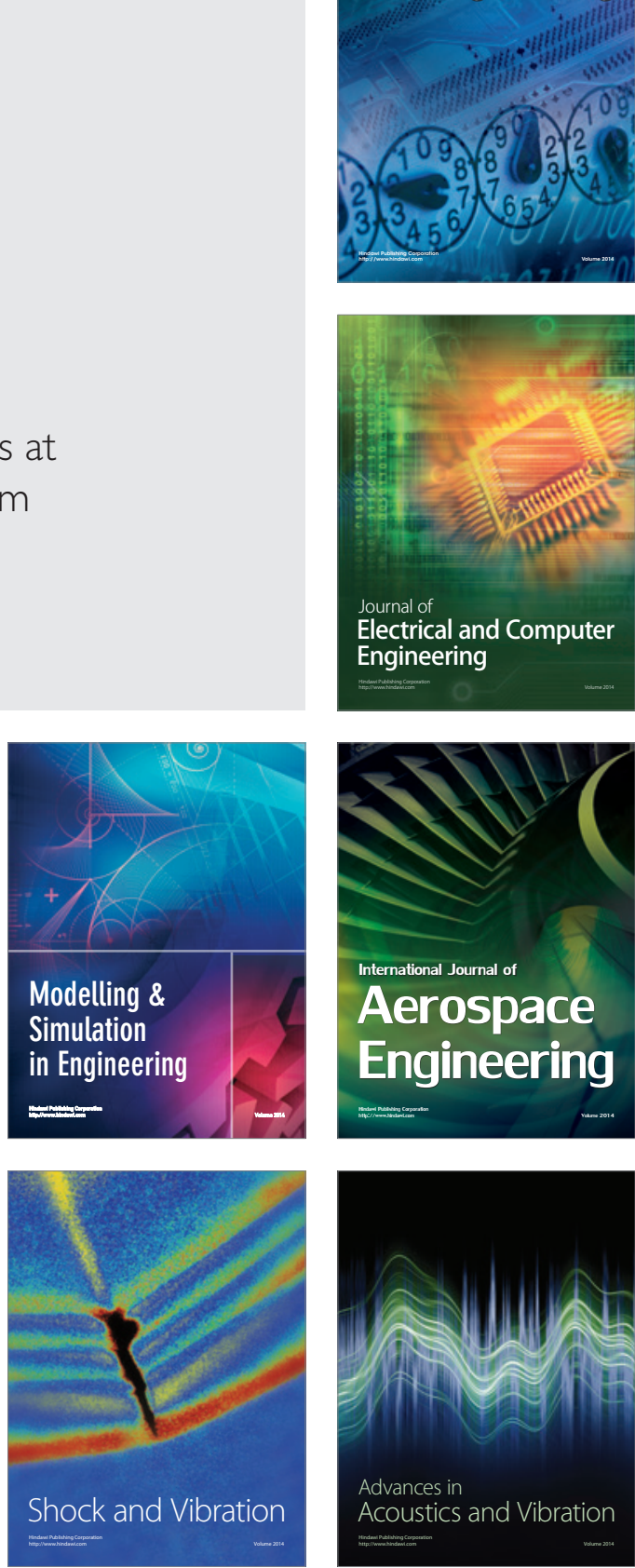